\title{
Dosimetric evaluation of vaginal cuff brachytherapy planning in cervical and endometrial cancer patients
}

\author{
Katarzyna Sikorska, MScl, Agnieszka Zolciak-Siwinska, PhD², Adam Kowalczyk, MScl, Michał Bijok, MSc', \\ Wojciech Michalski, MSc³, Ewelina Gruszczynska, MScl \\ 'Department of Medical Physics, Maria Sklodowska-Curie National Research Institute of Oncology, Warsaw, Poland, ${ }^{2}$ Department of \\ Gynecological Oncology, Maria Sklodowska-Curie National Research Institute of Oncology, Warsaw, Poland, ${ }^{3}$ Department of Clinical Trials \\ and Biostatistics, Maria Sklodowska-Curie National Research Institute of Oncology, Warsaw, Poland
}

\begin{abstract}
Purpose: The aim of the study was to perform a prospective analysis of dosimetric consequences of rectal enema administration before vaginal cuff brachytherapy (VCB), the dose distribution in organs at risk (OARs), and the presence of air gaps (AGs) in patients with cervical or endometrial cancer.

Material and methods: In total, 75 patients treated in 2019 were randomly divided into two groups including 38 patients with and 37 without an enema before VCB. All patients received post-operative high-dose-rate (HDR). Single-channel vaginal cylinders with active length of $2.75 \mathrm{~cm}$ were used. Prescription dose was $7 \mathrm{~Gy}$ at $5 \mathrm{~mm}$ depth from the applicator surface in all directions. Treatment plans were based on computed tomography (CT).

Results: Enema performed before cylinder insertion had no effect on rectosigmoid $\mathrm{D}_{\max }$ or $\mathrm{D}_{2 \mathrm{~cm} 3}$. Rectosigmoid median $\mathrm{V}_{100}$ was $0.5 \mathrm{~cm}^{3}$ (range, $0-2.7 \mathrm{~cm}^{3}$ ). $\mathrm{V}_{100} \geq 1 \mathrm{~cm}^{3}$ in 22 and $\geq 2 \mathrm{~cm}^{3}$ in 6 patients, with $\mathrm{D}_{\max }$ up to $19.7 \mathrm{~Gy}(282 \%$ ) were observed. No effect of bladder volume in the range of $27-256 \mathrm{~cm}^{3}$ on $D_{\max }$ or $\mathrm{D}_{2 \mathrm{~cm} 3}$ was found. The median bladder $\mathrm{V}_{100}$ was $0.1 \mathrm{~cm}^{3}$ (range, $0-1.4 \mathrm{~cm}^{3}$ ). There were $62(83 \%)$ patients with $\mathrm{AGs}$, with $24 \%$ at the top of the vagina and $75 \%$ on the remaining length of the vagina. Most of the AGs were small $(\leq 3 \mathrm{~mm})$, but in $5(8 \%)$ cases, they were bigger than $5 \mathrm{~mm}$.

Conclusions: VCB planning with the use of CT is essential. CT can facilitate the selection of optimal cylinder size to reduce the occurrence of large AGs. A few percent of plans require correction of dose distribution because of hot spots in OARs and the presence of AGs. Enema before cylinder insertion does not influence rectosigmoid $\mathrm{D}_{\max }$ and $\mathrm{D}_{2 \mathrm{~cm} 3}$. The analysis revealed no bladder volume effect on bladder doses $D_{\max }$ and $D_{2 \mathrm{~cm} 3}$.

J Contemp Brachytherapy 2020; 12, 3: 248-251 DOI: https://doi.org/10.5114/jcb.2020.96865
\end{abstract}

Key words: HDR, CT, VCB, cervical or endometrial cancer, enema, AGs.

\section{Purpose}

The use of computed tomography (CT) in delineation of organs at risk (OARs) and target volumes is wellknown. Vaginal cuff brachytherapy (VCB) is adjuvant treatment delivered after hysterectomy in cervical and endometrial cancer patients, alone or combined with external beam irradiation [1]. The primary purpose of the present study was to prospectively investigate the dosimetric consequences of administering rectal enemas prior to VCB. The secondary objective was to analyze the dose distribution in OARs, using standard planning with multiple points optimization $5 \mathrm{~mm}$ from the applicator surface such as 2D plans. The last goal was to record the presence of air gaps (AGs), which can potentially influence the treatment outcome.

\begin{abstract}
Material and methods
In 2019, 75 consecutive patients with indications for post-operative VCB for endometrial or cervical cancer were recruited. Sixty-two patients with endometrial cancer and 13 patients with cervical cancer were treated. In 35 cases, VCB was the only adjuvant treatment. In 40 patients, post-operative treatment was composed of external beam irradiation and brachytherapy. All patients received post-operative high-dose-rate (HDR) VCB in an outpatient setting. Phosphate enema was performed in 38 patients, and 37 were not specially prepared before the applicator insertion. Patients who had an enema before the VCB were selected randomly. The patients were asked to empty their bladder before the procedure. Single-channel vaginal cylinders of the largest diameter
\end{abstract}


$(2-3.5 \mathrm{~cm})$ that could fit comfortably inside the vagina vault were used. The applicators were placed while asking the patients to relax and breathe, because it is essential to use the largest possible cylinders for good coverage of vaginal mucosal tissue. The following cylinder diameters were used for the procedure: $2.0 \mathrm{~cm}$ (2 patients), $2.5 \mathrm{~cm}$ (32 patients), $3.0 \mathrm{~cm}$ (40 patients), and $3.5 \mathrm{~cm}$ (1 patient).

Pelvic CT scans were carried out in the supine position, with $3 \mathrm{~mm}$ slice thickness and no gap between slices. CT images were transferred to a 3D treatment planning system (Oncentra Brachy v.4.5.3, Elekta, Sweden). Organs at risk were contoured by the radiation oncologist on the consecutive CT slices according to recommendations [2]. We assessed the dose in rectum and sigmoid as the one-organ rectosigmoid. The outer bladder wall and the rectosigmoid from $1 \mathrm{~cm}$ over the cylinder tip to the anus were delineated. An active length of $2.75 \mathrm{~cm}$ (11 activated dwell source positions) was used and optimized to deliver a fraction dose of 7 Gy at $5 \mathrm{~mm}$ depth from the applicator surface in lateral and cranial directions. Patients were planned in accordance with the GEC-ESTRO handbook of brachytherapy, as presented in Figure 1. For a purpose of the study, we analyzed measurements for one 7 Gy fraction. Dose-volume histograms (DVHs) were generated. Brachytherapy (BT) was given to all patients using an iridium-192 ( $\left.{ }^{192} \mathrm{Ir}\right)$ source, with an initial nominal specific activity of $10 \mathrm{Ci}$ using the MicroSelectron HDR afterloading system (Elekta). The protocol and consent procedure were approved by the local medical authority.

For the purpose of this analysis, all plans were created according to the GEC-ESTRO recommendations without dose corrections - standard plan. In cases of very high doses in OARs or AGs over $5 \mathrm{~mm}$ in irradiated areas, we corrected the treatment plans individually for each patient. Decision was made by a physician based on the treatment history of patient. All air gaps were recorded in the whole length of vagina.

\section{Statistical analysis}

Descriptive statistics were used to describe and analyze a specific dataset, providing short summaries of the sample and measures of central tendency (mean, median, and mode), and measures of variability (standard deviation, variance, minimum and maximum values). The sets and distribution of our data were analyzed, and histograms of all variables were plotted. Distributions of all variables, except for the bladder volume, were considered

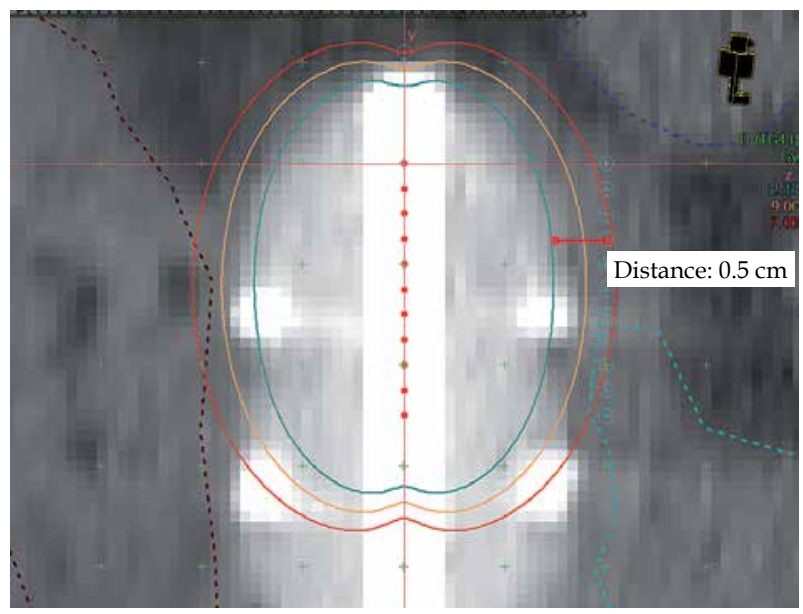

Fig. 1. Doses distribution around vaginal cylinder applicator with multiple points used for optimization

Brown - rectosigmoid, Dark blue - intestine, Light blue - bladder. Blue 259 circles are points used for optimization, Distance $-0.5 \mathrm{~cm}$

as a normal distribution. The comparative analysis was performed using Student's $t$-test (enema vs. rectal dose). Then, correlation analysis (non-parametric correlation, Spearman's test) and analysis of variance (ANOVA) were used for bladder volume vs. bladder doses. The whole group (with and without enemas) was divided into three equal sets according to the variable determining the volume of bladder.

\section{Results}

The median age of patients was 62 years (range, 3585 years). Data derived from DVHs for the whole group $(n=75)$ are presented in Table 1.

Enema was performed in 38 patients. In 37 cases, CT was completed without any special bowel preparation for the procedure. Enema before cylinder insertion did not influence rectosigmoid $\mathrm{D}_{\max }(p=0.38)$ and $\mathrm{D}_{2 \mathrm{~cm} 3}$ (the minimum dose to the highest treated $2 \mathrm{~cm}^{3}, p=0.056$ ) (Figure 2). Data showing mean doses for rectosigmoid with and without enema are given in Table 2.

The bladder volume in the volume range of $27-256 \mathrm{~cm}^{3}$ was not associated with an increase in $\mathrm{D}_{\max }(p=0.77)$ or $\mathrm{D}_{2 \mathrm{~cm} 3}(p=0.181)$ in Student's t-test. Bladder $\mathrm{D}_{2 \mathrm{~cm} 3}$ vs. bladder volume are presented in Figure 3. In the second analysis, bladder volumes were divided into three equally numerous groups: $<46 \mathrm{~cm}^{3}, 46-84 \mathrm{~cm}^{3}$, and $>84 \mathrm{~cm}^{3}$,

Table 1. Doses given for organs at risk for the whole group of patients $(n=75)$

\begin{tabular}{|c|c|c|c|c|}
\hline Organ at risk & & Mean & Values range & Median \\
\hline Rectosigmoid $D_{2 \mathrm{~cm}^{3}}$ & [Gy] & 5.8 (SD 0.8) & $3.5-7.4$ & 5.7 \\
\hline \multirow[t]{2}{*}{ Rectosigmoid $D_{\max }$} & {$[G y]$} & 9.3 (SD 1.9) & $5.8-19.7$ & 8.8 \\
\hline & {$[\%]$} & $132.9($ SD 28.1) & $82.3-282$ & 126.2 \\
\hline Bladder $\mathrm{D}_{2 \mathrm{~cm}^{3}}$ & {$[G y]$} & 4.9 (SD 0.9) & $2.3-6.7$ & 4.9 \\
\hline \multirow[t]{2}{*}{ Bladder $D_{\max }$} & [Gy] & 7.7 (SD 1.5) & $3.4-11.0$ & 7.9 \\
\hline & {$[\%]$} & 109.9 (SD 21.7) & $48.5-157.9$ & 113 \\
\hline
\end{tabular}

$S D$ - standard deviation, $D_{2 \mathrm{~cm} 3}$ - minimum dose to the highest treated $2 \mathrm{~cm}^{3}$ of the organ, $D_{\text {max }}$ - maximum dose to the whole organ 


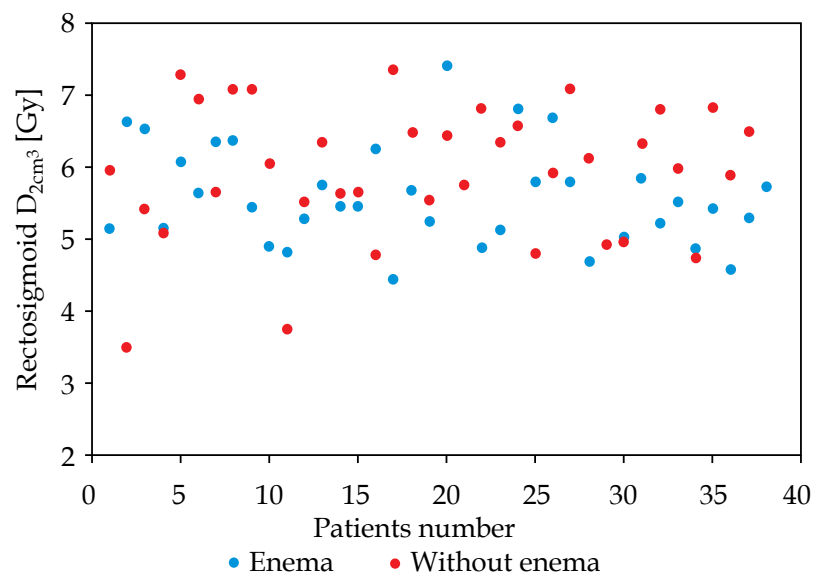

Fig. 2. Presentation of rectosigmoid dose in $D_{2 \mathrm{~cm} 3}$ with and without enema

for a comparison. There were no bladder volume effect on bladder doses noted, $\mathrm{D}_{2 \mathrm{~cm}^{3}}(p=0.597)$ and $\mathrm{D}_{\max }(p=0.229)$.

The volume of organs at risk covered by the prescribed dose $V_{100}$ was assessed. The median bladder $V_{100}$ was $0.1 \mathrm{~cm}^{3}$ (range, $0-1.4 \mathrm{~cm}^{3}$ ). Rectosigmoid median $\mathrm{V}_{100}$ was $0.5 \mathrm{~cm}^{3}$ (range, $0-2.7 \mathrm{~cm}^{3}$ ). There were 22 patients with $\mathrm{V}_{100}$ bigger than $1 \mathrm{~cm}^{3}$ and 6 patients with $\mathrm{V}_{100}$ bigger than $2 \mathrm{~cm}^{3}$ in rectosigmoid. These 6 patients with $V_{100}$ $\geq 2 \mathrm{~cm}^{3}$ had a $D_{2 \mathrm{~cm} 3}$ doses in the range of 7.1-7.4 Gy and $\mathrm{D}_{\max }$ in the range from $9.7 \mathrm{~Gy}(139 \%)$ to $19.7 \mathrm{~Gy}(282 \%)$.

There were $62(83 \%)$ patients with air gaps around the cylinder in the whole group. In 15 (24\%) cases, AGs were located at the apex (upper $3 \mathrm{~cm}$ of cylinder including the top of the vault) and in 47 (75\%) patients, AGs were situated in the lower part of vagina. The AGs depth was smaller than $3 \mathrm{~mm}$ in $37(59 \%)$ cases and bigger than $3 \mathrm{~mm}$ in 25 (40\%) patients. The median thickness of AGs was $3 \mathrm{~mm}$ (range, 2-6.5 mm). There were $5(8 \%)$ cases with AGs bigger than $5 \mathrm{~mm}$, one in the apex of vagina, and 4 within 46-57 $\mathrm{mm}$ below the apex. When $7 \mathrm{~Gy}$ was delivered at $5 \mathrm{~mm}$ depth from the applicator surface, the dose on vaginal mucosa was about 12.4 Gy. In case of an AG appearance with $3 \mathrm{~mm}$ depth, the dose decreased to $9 \mathrm{~Gy}$.

\section{Discussion}

In our study, enema performed before cylinder insertion in 38 patients did not influence rectosigmoid $D_{\max }$ and $\mathrm{D}_{2 \mathrm{~cm} 3}$. The enema administration could potentially reduce the rectal dose by reducing the rectal volume.

In literature, results of observations are indecisive. In one study, authors reported that larger rectal volumes were associated with higher rectal dose parameters during VCB [3]. Unfortunately, we did not find a relationship between preparation for the procedure with enema and higher doses in rectosigmoid, which was also confirmed by other authors $[4,5]$. However, a reduction of rectal doses can be achieved by removal of gas in the rectum during VCB [6]. A significant decrease of the mean rectum volume by $29 \%$ and $D_{2 \mathrm{~cm} 3}$ by $11 \%$ was observed by Sabater et al. [6]. In our study, we did not measure gas volume in rectosigmoid. The weak point of our study is
Table 2. Mean doses to rectosigmoid for patients with enema vs. without enema

\begin{tabular}{lcc} 
& $\begin{array}{c}\text { Enema } \\
(n=38)\end{array}$ & $\begin{array}{c}\text { Without enema } \\
(n=37)\end{array}$ \\
\hline Rectosigmoid $\mathrm{D}_{2 \mathrm{~cm}^{3}}[\mathrm{~Gy}]$ & $5.6(\mathrm{SD} 0.7)$ & $5.9(\mathrm{SD} 0.9)$ \\
\hline Rectosigmoid $\mathrm{D}_{\max }[\%]$ & $130.0(\mathrm{SD} 30.3)$ & $135.8(\mathrm{SD} 25.6)$
\end{tabular}

$S D$ - standard deviation, $D_{2 \mathrm{~cm} 3}$ - minimum dose to the highest treated $2 \mathrm{~cm}^{3}$ of the organ, $D_{\max }-$ maximum dose to the whole organ

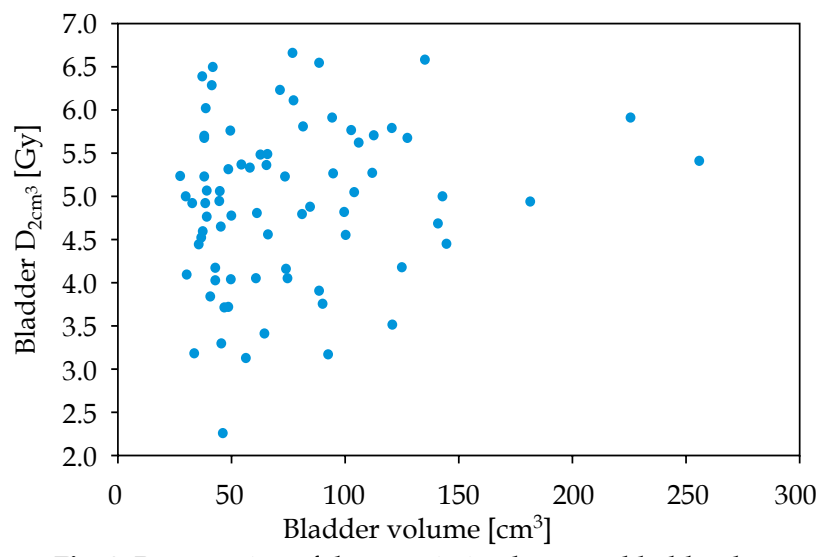

Fig. 3. Presentation of the association between bladder dose in $2 \mathrm{~cm}^{3}$ and bladder volume

the lack of CT planning carried out with and without enema in the same patient.

The bladder volume in the range of $27-256 \mathrm{~cm}^{3}$ did not influence $\mathrm{D}_{\max }$ or $\mathrm{D}_{2 \mathrm{~cm} 3}$ in our study. Literature data on the analysis of the effect of bladder volume on the dose given during VCB are also inconclusive. In a dosimetric study, Mahantshelly et al. compared the mean bladder volumes of $64.5 \mathrm{~cm}^{3}, 116.2 \mathrm{~cm}^{3}$, and $172.9 \mathrm{~cm}^{3}$, and a significant increase in small bowel dose in the empty bladder when compared to the full bladder was noted for intracavitary BT in cervical cancer patients. Only non-significantly higher bladder doses in 3 series were observed [7]. Like in our study, bladder filling did not increase the dose in it. The dosimetric effects of bladder filling in patients with VCB with a distended bladder preferentially reduce high-dose to the small bowel around the vaginal cuff without a significant change in dose to the bladder, rectum, or sigmoid, as reported by Hung et al. [8]. On the other hand, in literature, the full bladder produces a significant, $18.7 \%$ of bladder $\mathrm{D}_{2 \mathrm{~cm} 3}$ increase [9], and an empty bladder in 35 of 45 women reduced bladder doses by 0.5 Gy on average [10]. Hoskin et al. analyzed doses in empty and three full bladder volumes $(35,70$, and $100 \mathrm{ml})$, and showed that the maximum bladder dose was lower with the empty bladder than with any of the full ones [11].

The analysis of $D_{\max }$ and $V_{100}$ in OARs is very important. The analysis of $\mathrm{V}_{100}$ in the rectosigmoid revealed large differences in our study. $\mathrm{A}_{2 \mathrm{~cm} 3}$ dose in the rectosigmoid equal to 7.4 Gy was acceptable, but $D_{\max }=19.7 \mathrm{~Gy}$ was not. There is no recommendation concerning $\mathrm{D}_{\max }$ and $\mathrm{V}_{100}$ in OARs in the literature; however, the $\mathrm{V}_{100}$ or $\mathrm{D}_{\max }$ reduction in cases on overdosage is required. Our 
data based on standard plans, emphasized the benefit of 3D over 2D planning.

This study analyzed doses in the rectosigmoid, not only in the rectum, and it is a possible reason for the slightly higher dose in the organ. We decided not to contour these two organs (rectum and sigmoid) separately due to a short length of source position activation in the cylinder $(2.75 \mathrm{~cm})$, to avoid overdosage of OARs. High doses in OARs are observed in patients with thin vaginal walls and coexistent bowel loops adjacent to the apex of vagina. In such cases, it can be considered to prescribe the dose to the applicator surface, not at $0.5 \mathrm{~cm}$ depth. The utility of repeated OARs dose-volume histogram calculations in multifractional HDR VCB, using 3-dimensional imaging was assessed by Holloway et al. [12]. Variation of within-patients coefficients of $\mathrm{D}_{0.1 \mathrm{~cm} 3}$ and $\mathrm{D}_{2 \mathrm{~cm} 3}$ were for the bladder: $14 \%$ and $8.1 \%$, for the rectum: $7.9 \%$ and $5.9 \%$, and for the sigmoid: $27.6 \%$ and $20.3 \%$, respectively. The authors in [12] concluded that the small variation in doses to the bladder and rectum did not support reporting doses to the OARs beyond the initial fraction. In our study, CT was performed only before the first brachytherapy fraction.

Identification of AGs in our material was $83 \%$ in the whole length of the vagina, and $24 \%$ of AGs were located in the upper $3 \mathrm{~cm}$ of the vagina, including the top of the vault. In a meta-analysis of 9 publications, which met the requirements, $67 \%$ of patients had at least one air pocket and $59 \%$ per insertion [13]. Sapienza et al. in a prospective study reported that $77.2 \%$ of AGs were located within the proximal $2 \mathrm{~cm}$ of the cylinder, and $81.8 \%$ within the proximal $4 \mathrm{~cm}$ of the cylinder [13].

The depth of AGs is essential. In our study, $59 \%$ of AGs were small ( $\leq 3 \mathrm{~mm})$, allowing the accepted dose to be given to the vaginal mucosa. In one patient, a $6 \mathrm{~mm}$ AG was located in the apex of vagina, adversely influencing dose distribution.

The incidence of AGs depends on the cylinder diameter, the proper fixation, and relaxation during the procedure. AGs reduce the mucosal dose in the treated area. Since the presence of AGs is often found, post-insertion CT can facilitate selection of optimal cylinder size in VCB or allow modification of dose distribution while planning the process. In our material, only some AGs were observed at the apex of the vagina (upper $3 \mathrm{~cm}$ ).

\section{Conclusions}

Vaginal cuff brachytherapy planning with the use of CT is essential. Enema before cylinder insertion does not influence rectosigmoid $\mathrm{D}_{\max }$ and $\mathrm{D}_{2 \mathrm{~cm} 3}$. The analysis revealed no bladder volume effect on bladder doses $D_{\max }$ and $\mathrm{D}_{2 \mathrm{~cm} 3}$. A few percent of plans require correction of dose distribution because of hot spots in OARs. For patients with high $\mathrm{D}_{\max }$ and $\mathrm{V}_{100}$ in OARs, it should be considered to prescribe the dose to the applicator surface, or at smaller than $0.5 \mathrm{~cm}$ depth.

Most patients have air gaps around the cylinder, which can potentially reduce the mucosal dose. Selection of cylinder should be based on clinical findings. Post-insertion CT can facilitate a selection of optimal cylinder size. In case of major AGs, dose variation optimization of dose distribution is required. AGs less than $3 \mathrm{~mm}$ can be disregarded.

\section{Disclosure}

The authors report no conflict of interest.

\section{References}

1. Small W, Beriwal S, Demanes DJ et al. American Brachytherapy Society consensus guidelines for adjuvant vaginal cuff brachytherapy after hysterectomy. Brachytherapy 2012; 11: 58-67.

2. Pötter R, Haie-Meder C, Van Limbergen E et al.; GEC ESTRO Working Group. Recommendations from gynaecological (GYN) GEC ESTRO working group (II): concepts and terms in 3D image-based treatment planning in cervix cancer brachytherapy - 3D dose volume parameters and aspects of 3D image-based anatomy, radiation physics, radiobiology. Radiother Oncol 2006; 78: 67-77.

3. Sabater S, Arenas M, Berenguer R et al. Dosimetric analysis of rectal filling on rectal doses during vaginal cuff brachytherapy. Brachytherapy 2015; 14: 458-463.

4. Sabater S, Andres I, Gascon M et al. Effect of rectal enemas on rectal dosimetric parameters during high-dose-rate vaginal cuff brachytherapy. Strahlender Oncol 2016; 192: 248-253.

5. Andres I, Gutiemez-Perez M, Rodrigez-Vela MF et al. The usefulness of fleet rectal enemas on high-dose-rate intracavitary cervical cancer brachytherapy. A prospective trial. J Contemp Brachytherapy 2017; 9: 224-229.

6. Sabater S, Sevillano MM, Andres I et al. Reduction of rectal doses by removal of gas in the rectum during vaginal cuff brachytherapy. Strahlenther Oncol 2013; 189: 951-956.

7. Mahantshetty U, Shetty S, Majumder D et al. Optimal bladder filling during high-dose-rate intracavitary brachytherapy for cervical cancer: a dosimetric study. J Contemp Brachytherapy 2017; 9: 112-117.

8. Hung J, Shen S, De Los Santos J et al. Image-based 3d treatment planning for vaginal cylinder brachytherapy: dosimetric effects of bladder filling on organs at risk. Int J Radiation Oncol Biol Phys 2012; 83: 980-985.

9. Guler OC, Onal C, Acibuci I. Effects of bladder distention on dose distribution of vaginal vault brachytherapy in patients with endometrial cancer. J Contemp Brachytherapy 2015; 6: 371-376.

10. Kobzda JD, Cikowska-Wozniak E, Michalska M et al. Three-dimensional dosimetry of the full and empty bladder in high dose rate vaginal cuff brachytherapy. Int J Gynecol Cancer 2014; 24: 923-927.

11. Hoskin PJ, Vidler K. Vaginal vault brachytherapy: the effect of varying bladder volumes on normal tissue dosimetry. $\mathrm{Br}$ J Radiol 2000; 73: 864-866.

12. Holloway CL, Maclin EA, Cormack RA et al. Should the organs at risk be contoured in vaginal cuff brachytherapy? Brachytherapy 2011; 10: 313-317.

13. Sapienza LG, Ning MS, Cassio de Assis Pellizzon A et al. Detection of air gaps around the cylinder by postinsertion computed tomography in vaginal cuff brachytherapy: A prospective series, systematic review, and metaanalysis. Brachytherapy 2019; 18: 620-626. 